\title{
ARQAS: Augmented Reality based Question Answering System using Ontology in HINDI and MARATHI Language
}

\author{
Dhiraj Amin \\ Department of Computer Engineering \\ PIIT, New Panvel, India
}

\author{
Sharvari Govilkar \\ Department of Computer Engineering \\ PIIT, New Panvel, India
}

\begin{abstract}
Question answering (QA) system helps user to find the precise answer of the question articulated in natural language. The most important part of QA system is how the answers are presented to the users where not only how much relevant answer retrieved for the question is important but also how these answers are presented to the users is also important. Augmented reality (AR) can be used as platform or interface to enrich the way how the answers are presented of the questions, where user can feel to be a part of that information deduced from the answer. We have proposed a system where users can ask query in the form of natural language and get relevant information to their queries in the form of different virtual content augmented in their real environment. Semantic matching for user natural language query has been performed efficiently by using ontology. We have limited this work to Hindi and Marathi language.
\end{abstract}

\section{General Terms}

Question answering, Information retrieval, Natural Language Processing, Augmented Reality, Information Extraction.

\section{Keywords}

Augmented Reality QA, Question answering system (QAS), Question answering (QA), Ontology, Augmented, Augmented Reality (AR), Natural language question answering system (NLQA), Augmented Reality based Question Answering system (ARQAS), Natural language processing (NLP).

\section{INTRODUCTION}

Question answering system (QAS) provides precise answer to question provided by the users. Most of the questions in QAS are in the form of natural language query, where user has freedom to provide their questions in their own natural language. A question answering system using semantic matching (achieved using ontology) provides more accurate answers. Ontology is a form of conceptual representation of information where relation between different entity and details about a particular entity is provided.

The most important part of QA system is how the answers are presented to the users, as questions are asked to extract answers so it can be clearly inferred as; answers and its representation is the most important part in such system. Not only how much relevant answer retrieved for the question is important but also how these answers are presented to the users is important. Most of the QA system presents answers in text form or sometimes image, but understanding of answers by users can be easier, when answers are presented in their augmented environment where they can feel to be part of those answers. This can be achieved by using augmented reality as platform or interface to present answers to the questions.

The paper presents, an augmented reality based question answering system in Hindi and Marathi language where answers to the user questions are presented in user augmented environment. In section 2, proposed augmented reality based question answering system using ontology (ARQAS) is discussed in detail. Working of system is mentioned in detail in section 3. Section 4 explores accuracy obtained by ARQAS. Finally, paper is concluded in section 5.

\section{PROPOSED SYSTEM}

We propose a system titled augmented reality based question answering system using ontology (ARQAS) which provides a unique way of presenting answer of users question in augmented environment where user can feel to be part of answer. This system provides the answer to the question present in user mind through use of natural language processing to process question to retrieve accurate answer and augmented reality to present answer in augmented environment of user. The input to the system is user question in Hindi or Marathi language and output will be augmentation of required answer in the user's augmented environment, where user can feel to be part of the answers in real time.

The proposed system consists of two modules:

1. Question Answering

\section{Augmented Reality}

Figure 1 presents architecture of our system. Here first, user specifies the query in natural language i.e. Hindi or Marathi language. User can specify query in the form of speech or text for Hindi language and textual for Marathi language.

In question answering module, input query is first tokenized into separate tokens and word grouping is performed on tokenized query considering bigrams and trigrams tokens. Tokenized input query is then provided to Part of Speech tagger (POS) which assigns part of speech to respective token. POS tagged query text is then chunked as per noun and verb chunking rules. From the chunked query text, first type of question is extracted then subject, object and verb/predicate are extracted based on Subject, Object, and Verb (SOV) extraction rule. Using extracted SOV and question type, query triples are generated which are then converted into onto triples by extracting respective onto words from the ontology. Finally ontology is traversed where onto triples are matched with concept/entity in ontology to retrieve the relevant answer index. 
In augmented reality module, from the extracted answer index corresponding 2D/3D virtual objects are retrieved and augmented with the real environment of the user.

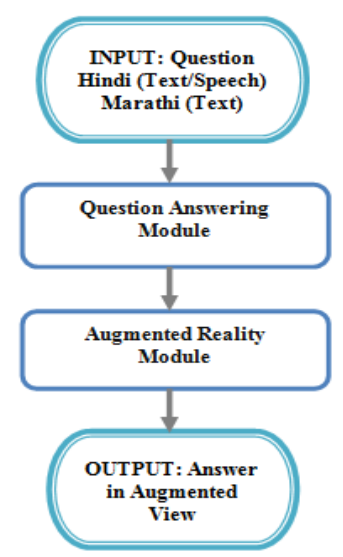

Figure 1: Proposed System

\section{WORKING OF SYSTEM}

ARQAS combines a standalone question answering system with augmented reality to enrich the way of presenting information to the users.

ARQAS is created as an android application with the help of Metaio SDK [5] to support for augmented reality. Augmented Reality SDK facilitates many components within the AR application: Augmented reality tracking, Augmented reality recognition and Augmented reality content rendering [1]. On basis of our past survey [1] we found that Metaio SDK was best suited for our application development due its simplicity and better tracking quality and types compared to other augmented reality SDK.

An ARQAS supports natural language query in Hindi and Marathi language. With the help of Google Speech to Text [2][3] functionality user can ask questions to our system in Hindi language in the form of natural language speech.

User can provide same query in different word order, as in Hindi and Marathi language subject, object and verb can come in any order so a same query can be asked to our system by reformulating it in different way and still we get relevant answers. Following natural language query can be formulated in different way:

Hindi Query

Form 1: सौर मंडल में सबसे विशाल ग्रह कोनसा है?

Form 2: सौर मंडल में कोनसा सबसे विशाल ग्रह है?

Form 3: सबसे विशाल ग्रह कोनसा है सौर मंडल में?

Marathi Query

Form 1: सूर्यमालामध्ये सर्वात विशाल ग्रह कोणता आहे?

Form 2: सूर्यमालामध्ये कोणता सर्वात विशाल ग्रह आहे?

Form 3: सर्वात विशाल ग्रह कोणता आहे सूर्यमालामध्ये?

ARQAS is based on Metaio image tracking functionality [4] which is a sort of marker less tracking which tracks planar objects like poster or printed image. Figure 2 Shows image tracker used for our system. Any form of image which rich visual details can set as picture marker. Virtual content to be displayed to the users as answers of their question are augmented on this image. So if image is not present in the field of view of device camera then virtual content won't be augmented in user real environment.

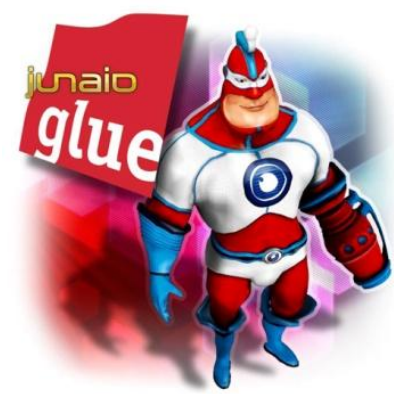

Figure 2: Image tracker used in ARQAS

Figure 3 demonstrate user interface of our system. Here user has option to rotate virtual content, scale content to different sizes and also translate so that content can be adjusted nearer or farther from users.

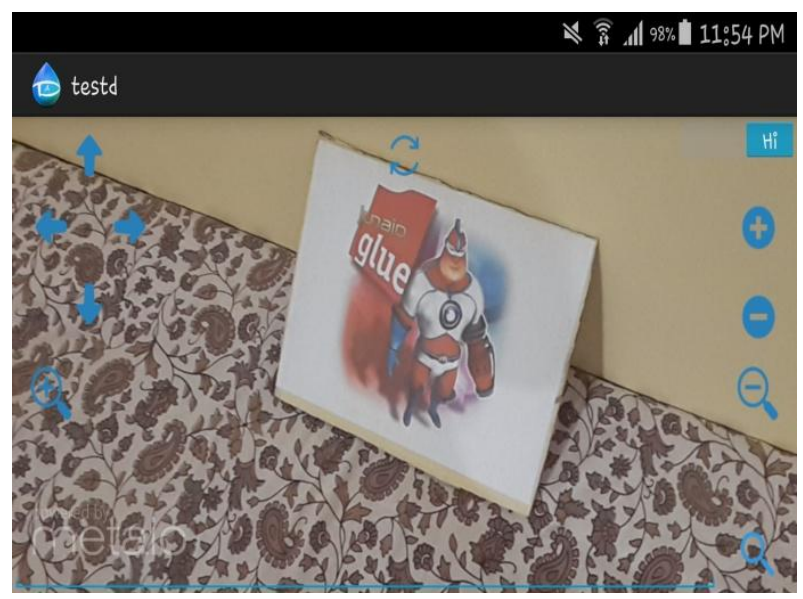

Figure 3: User interface of proposed ARQAS

Figure 4 Demonstrate flow of our system. Initially when the application starts tracking configuration is loaded which contains details about image to be tracked and configuration for setting up target. Then all the virtual contents are loaded.

After query is provided by user then based on the language selected, users question text is passed onto respective Hindi or Marathi question answering module (containing tokenization, word grouping, POS tagging, Chunking, Query Triple extraction, Onto Triple Extraction, Onto matching and fetching of answer) which generates answer index to be passed to augmented reality module to fetch and augment virtual contents in user real environment.

Hindi Question: सौर मंडल में सबसे विशाल ग्रह कोनसा है?

Marathi Question: सूर्यमालामध्ये सर्वात विशाल ग्रह कोणता आहे?

In question answering module first the query text is tokenized into tokens. Then neighboring words which can represent as a single entity are grouped together. 


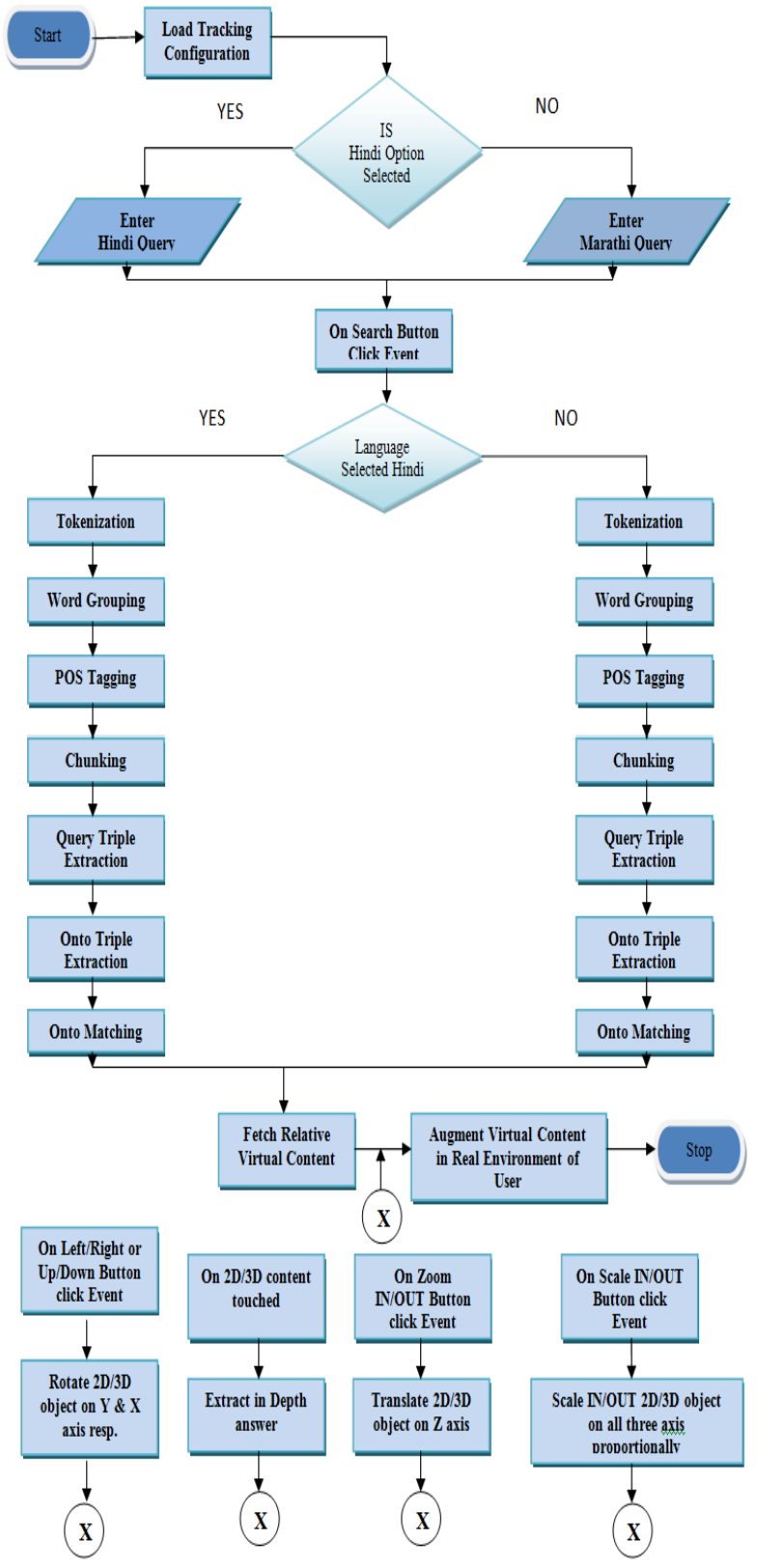

Figure 4: Flow chart of ARQAS

Word Grouped Tokenized Query:

\section{Hindi}

Token 1: सौर_मंडल

Token 2: में

Token 3: सबसे

Token 4: विशाल

Token 5: ग्रह

Token 6: कोनसा

Token 7: है
POS tagging is performed for each token of the query text where part of speech like Noun, Verb, Adjective, etc. are assigned to the words.

POS tagged query:

$$
\text { Hindi Marathi }
$$

सौर_मंडल::NNP

सूर्यमालामध्ये::NNP

में::CM सर्वात::INTF

सबसे::INTF

विशाल::JJ

विशाल::JJ

ग्रहः:NN

ग्रह::NN

कोणता::WQ

कोनसाः:WQ

आहे::VAUX

\section{है::VAUX}

Chunking is performed to identify different proper noun, common noun and verb groups in query text.

Chunked query:

For Hindi Question

सौर_मंडल NNP में CM::NNPG

सबसे INTF विशाल $\mathrm{JJ}$ ग्रह $\mathrm{NN}:: \mathrm{NNG}$

कोनसा::WQ

है::VAUX

For Marathi Question

सूर्यमालामध्ये NNP::NNPG

सर्वात INTF विशाल JJ ग्रह NN::NNG

कोणताः:WQ

आहे::VAUX

Then in query triple extraction query triplets are extracted from query text by using extracted SOV.

Query Triple:

For Hindi Question

कोनसा(सौर_मंडल,सबसे_विशाल_ग्रह)

For Marathi Question

कोणता(सूर्यमाला,सर्वात_विशाल_ग्रह)

From the query triples corresponding onto triple are generated by replacing query term with appropriate onto term present inside ontology. And finally onto matching is performed by traversing ontology to retrieve relative answer index of the input query, if matched.

Onto Triple:

For Hindi Question

कौन(सौर_परिवार,सबसे_बड़ा_ग्रह) 
For Marathi Question

कोणते(सूर्यमाला,सर्वात_विशाल_ग्रह)

Once the relative answer index is extracted then corresponding virtual content is rendered on the user real view by augmenting that virtual content with the real environment of user.

For given Hindi and Marathi question, system retrieves answer index with value equals to 8 which then serves as input to display corresponding virtual content from the available content list.

Figure 5 Shows answer as 3D object of planet Jupiter (बृहस्पति or गुरू), as Jupiter is the largest planet in our solar system, for question "सौर मंडल में सबसे विशाल ग्रह कोनसा है?" in Hindi and question “सूर्यमालामध्ये सर्वात विशाल ग्रह कोणता आहे?" in Marathi.

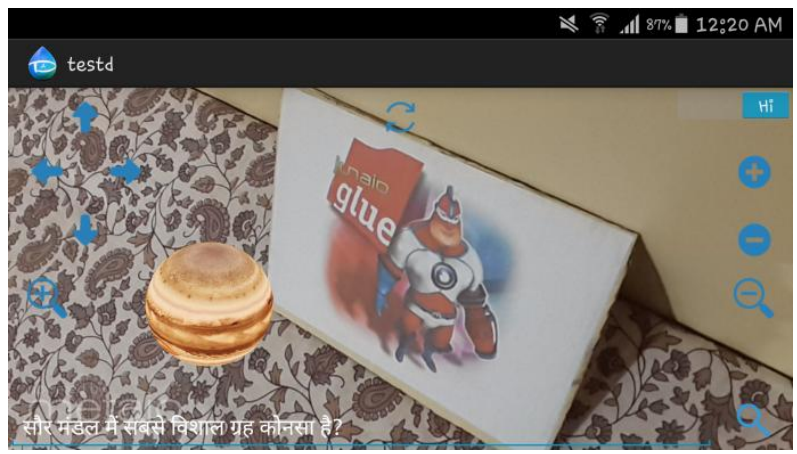

Figure 5: Output to user question.

As we have used Metaio image tracking, in our system virtual objects are augmented over the image present in the user physical world, this way a user experience is created where user can feel that answer to their query exist in reality nearer to them.

ARQAS also provides user to interact with virtual contents, where user can touch the virtual object to get more details about the answer of user query. Also one can rotate virtual objects on $\mathrm{x}$ and $\mathrm{y}$ axis to see object from different viewing angles. Object can be zoomed IN/OUT so that object can be seen nearer/farther from user. Scale option is also available so that user can see content with minute details.

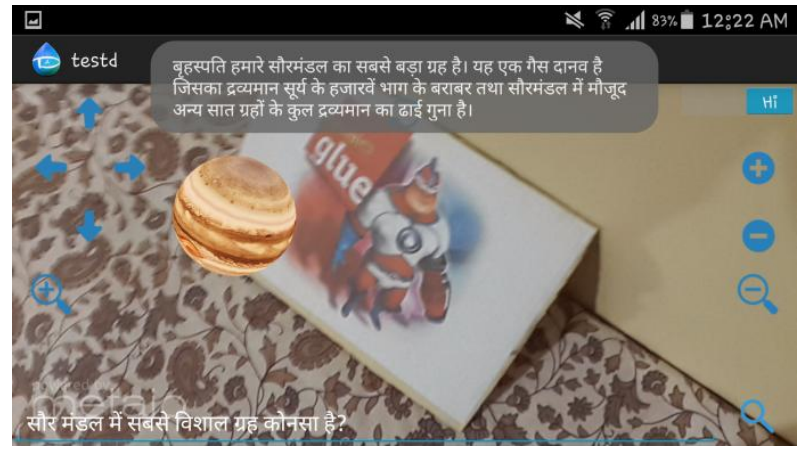

Figure 6: In depth answer on virtual object touched for

Hindi Query (“सौर मंडल में सबसे विशाल ग्रह कोनसा है?")

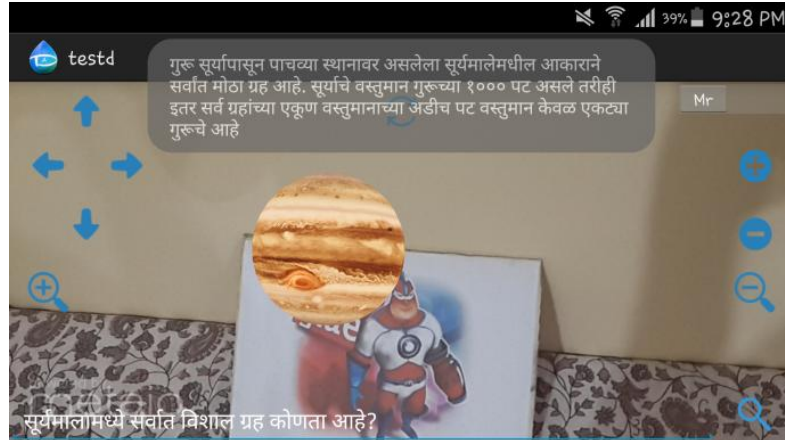

Figure 7: Detailed answer on virtual object touched for

Marathi Query ("सूर्यमालामध्ये सर्वात विशाल ग्रह कोणता

आहे?")

Figure 6 and Figure 7 Shows answer as 3D object of planet Jupiter for the input question with additional details of answer in small paragraph when user touches the Jupiter 3D object so that user gets more information regarding their question.

\section{PERFORMANCE ANALYSIS}

The system ARQAS is evaluated to check whether the answer generated for the user query is correct or not. To measure accuracy of system we have taken overall 40 Question comprising both of Hindi and Marathi language related to solar system. Out these 40 questions, 36 questions are valid question as per required question format. Finally out of those 36 valid question ARQAS could provide correct and relevant answer for 34 questions.

ARQAS show answers to most of the question from our question set. Some sample queries in Hindi and Marathi language for our system are listed below.

Table 1. Sample Queries for Hindi Language

\begin{tabular}{|c|l|}
\hline $\begin{array}{c}\text { Question } \\
\text { No. }\end{array}$ & \multicolumn{1}{c|}{ Question } \\
\hline 1 & सौर मंडल में क्या होता है? \\
\hline 2 & सौर मंडल में कितने विहग है? \\
\hline 3 & सौर मंडल में सबसे विशाल ग्रह कोनसा है? \\
\hline 4 & पृथ्वी के कितने प्राकृतिक उपग्रह है? \\
\hline 5 & पृथ्वी के निकटतम ग्रह कोनसा है? \\
\hline
\end{tabular}

Table 2. Sample Queries for Marathi Language

\begin{tabular}{|c|l|}
\hline $\begin{array}{c}\text { Question } \\
\text { No. }\end{array}$ & \multicolumn{1}{c|}{ Question } \\
\hline 1 & सूर्यमालामध्ये काय आहे? \\
\hline 2 & सूर्यमालामध्ये किती विहग आहे? \\
\hline 3 & $\begin{array}{l}\text { सूर्यमालामध्ये सर्वात विशाल ग्रह कोणता } \\
\text { आहे? }\end{array}$ \\
\hline 4 & पृथ्वीचे नैसर्गिक उपग्रह किती आहे? \\
\hline 5 & पृथ्वीचे निकटतम ग्रह कोणता आहे? \\
\hline
\end{tabular}


ARQAS gives accuracy of $94.4 \%$ for the valid questions asked by user based on the solar system ontology information provide to them. Some question like "सबसे विशाल कोनसा ग्रह है सौर मंडल में?" don't provide relevant answer, as here due to such ordering of question the phrase "सबसे विशाल ग्रह" is not extracted while chunking and thus no answer is found of such question. However ARQAS answers almost all type of question it supports.

\section{CONCLUSION}

A question answering system where augmented reality is used as a medium to present answer to the query highly improves the probability of user inferring knowledge from the answers. Semantic matching using concept of ontology help to provide more relevant answers than the traditional keyword based matching methods. AR offers methods of data enrichment through direct superimposition virtual objects onto the user physical world. With the help of AR as platform to present information to the user the QA system can mediate the answers as a part of user physical or real world. Natural language query's also eases out the task of users by providing the user to interact with the system in their daily spoken natural language.

Further ARQAS can be scaled to contain answers related to different domain and also support more Indian regional languages. Also different tracking functionality can be explored to render virtual content in real environment. ARQAS can become a very important system in education where students can infer knowledge from answer to their question very easily.

\section{REFERENCES}

[1] Dhiraj Amin and Sharvari Govilkar. "COMPARATIVE STUDY OF AUGMENTED REALITY SDK'S.", International Journal on Computational Sciences \& Applications (IJCSA) Vol.5, No.1, February 2015.

[2] Speech Recognizer, Available at: http://developer.android.com/reference/android/speech/S peechRecognizer.html (last accessed on June 13, 2015).

[3] Google Hindi Input, Available at: https://play.google.com/store/apps/details?id=com.googl e.android.apps.inputmethod.hindi\&hl=en (last accessed on June 13, 2015).

[4] Image Tracking, Available at: http://dev.metaio.com/sdk/tracking-config/opticaltracking/image-tracking/index.html (last accessed on June 13, 2015)

[5] Getting Started with the Metaio SDK, Available at: https://dev.metaio.com/sdk/ (last accessed on June 13, 2015). 